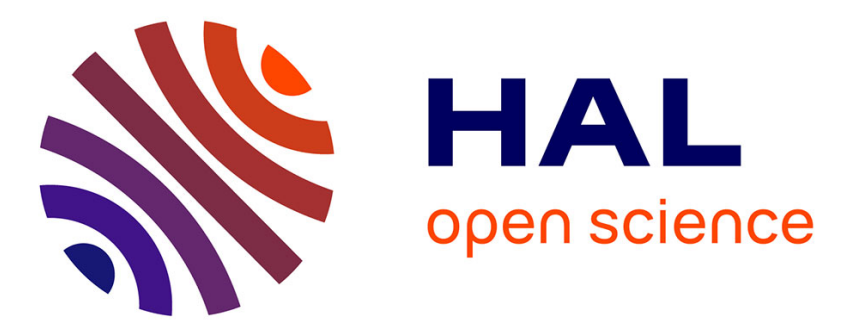

\title{
Approximate model for surface-plasmon generation at slit apertures
}

Philippe Lalanne, Jean-Paul Hugonin, Jean-Claude Rodier

\section{To cite this version:}

Philippe Lalanne, Jean-Paul Hugonin, Jean-Claude Rodier. Approximate model for surface-plasmon generation at slit apertures. Journal of the Optical Society of America. A Optics, Image Science, and Vision, 2006, 23 (7), pp.1608-1615. hal-00869602

\section{HAL Id: hal-00869602 \\ https://hal-iogs.archives-ouvertes.fr/hal-00869602}

Submitted on 3 Oct 2013

HAL is a multi-disciplinary open access archive for the deposit and dissemination of scientific research documents, whether they are published or not. The documents may come from teaching and research institutions in France or abroad, or from public or private research centers.
L'archive ouverte pluridisciplinaire HAL, est destinée au dépôt et à la diffusion de documents scientifiques de niveau recherche, publiés ou non, émanant des établissements d'enseignement et de recherche français ou étrangers, des laboratoires publics ou privés. 


\title{
Approximate model for surface-plasmon generation at slit apertures
}

\author{
P. Lalanne, J. P. Hugonin, and J. C. Rodier \\ Laboratoire Charles Fabry de l'Institut d'Optique, Centre National de la Recherche Scientifique, \\ F-91403 Orsay Cedex, France
}

\begin{abstract}
Received September 23, 2005; accepted November 10, 2005; posted January 10, 2006 (Doc. ID 64965)
We present a semianalytical model that quantitatively predicts the scattering of light by a single subwavelength slit in a thick metal screen. In contrast to previous theoretical works related to the transmission properties of the slit, the analysis emphasizes the generation of surface plasmons at the slit apertures. The model relies on a two-stage scattering mechanism, a purely geometric diffraction problem in the immediate vicinity of the slit aperture followed by the launching of a bounded surface-plasmon wave on the flat interfaces surrounding the aperture. By comparison with a full electromagnetic treatment, the model is shown to provide accurate formulas for the plasmonic generation strength coefficients, even for metals with a low conductivity. Limitations are outlined for large slit widths $(>\lambda)$ or oblique incidence $\left(>30^{\circ}\right)$ when the slit is illuminated by a plane wave. (C) 2006 Optical Society of America

OCIS codes: $050.1220,050.1940,260.2110,260.3910$.
\end{abstract}

\section{INTRODUCTION}

Because of the advent of nanotechnology and the recent applications of surface plasmon polaritons (SPPs) for manipulating light at a subwavelength scale, there has recently been reawakened interest in the physics governing the electromagnetic behavior of metal films perforated with subwavelength apertures for visible light operation. ${ }^{1-5}$ In that context, light scattering by an optically opaque metal film perforated by a single subwavelength aperture, like a hole or a slit, represents the more basic diffraction problem. Although it has been studied for a long time (see the early references ${ }^{6-8}$ ), this problem is still currently the subject of intense research from a theoretical point of view. Most of the earlier theoretical works relied on semianalytic or on intensive computational approaches ${ }^{9-12}$ of the near-field patterns and well reproduced or interpreted global experimental data such as the transmission through the aperture. However, they did not address the problem of the SPP generation at the input or output nanoaperture sides in a quantitative manner, although this generation has been evidenced experimentally through far-field measurements ${ }^{4,5}$ or direct near-field measurements performed on a single nanohole perforated in a thin metallic film. ${ }^{13,14}$ Recently, the SPP generation at a metallic interface perforated by an isolated subwavelength slit, when the slit is illuminated by its fundamental guided mode [Fig. 1(a)] or by an incident plane wave [Fig. 1(b)], has been tackled through a full electromagnetic treatment and a semianalytical model. ${ }^{15}$ However, due to space limitations, the model has been only briefly documented.

In this work we provide a detailed description of the model and quantitatively test its domain of validity. It is found that the model predictions are accurate even for noble metal with a low conductivity, as encountered in the visible region of the spectrum. In Section 2 we first present a theoretical formalism for the rigorous calcula- tion of the scattering coefficients between the incident light and the SPP modes launched at the slit aperture. This general formalism is rigorous in the sense that the scattering coefficients are computed with high accuracy. ${ }^{16}$ It represents an important outcome of this work that will be used to validate the model. Section 3 is devoted to the derivation of the semianalytical model. Approximate but accurate expressions for the SPP generation coefficients are obtained for the two scattering problems shown in Fig. 1. In Section 4, the model is applied to several geometries of interest in practice such as grooves in a metallic substrate. This allows us to discuss the influence of the different parameters of the scattering problem. Additionally, the model predictions are tested against data obtained with the theoretical formalism, which allows an indepth test for the complex amplitude scattering coefficients associated with the SPP generation mechanisms. Section 5 summarizes the main results.

Hereafter, the metal is considered as a real metal with a finite conductivity. Gold will be used to illustrate our discussion, and its frequency-dependent permittivity $\epsilon$ is taken from Ref. 17. Let us emphasize that although gold is used throughout the paper, the analysis and the obtained results remain valid for other metals.

\section{THEORETICAL FORMALISM}

Let us consider the geometry shown in Fig. 1(a). The slit has the same direction as the magnetic field $H_{y}$ (transverse-magnetic polarization) and is illuminated by its fundamental guided mode at a fixed wavelength $\lambda(k$ $=2 \pi / \lambda=\omega / c)$. In the figure, $n_{1}$ and $n_{2}$ refer to the refractive indices of the dielectric materials in the slit and below the slit. We denote the slit width by $w$. Inside the slit in medium 1, the magnetic field admits a modal expansion of the form 


$$
H_{1}(x, z)=\Psi_{0}(x) \exp \left(i k n_{0}^{\mathrm{eff}} z\right)+\Sigma_{p} r_{p} \Psi_{p}(x) \exp \left(-i k n_{p}^{\mathrm{eff}} z\right),
$$

where $p$ is an integer, $\Psi_{p}$ is the magnetic field of mode $p$, and $n_{p}^{\text {eff }}$ is its normalized propagation constant. The subscript 0 refers to the fundamental propagative mode with a weak attenuation $\operatorname{Im}\left(n_{0}^{\text {eff }}\right) \ll 1$. Similarly, below the slit in medium 2 , the magnetic field $H_{2}$ can be expanded into a plane-wave basis:

$$
H_{2}(x, z)=\int_{-\infty}^{\infty} \mathrm{d} u t_{u} \exp \left(i k n_{2} u x\right) \exp \left(i k n_{2} \gamma_{u} z\right)
$$

where $u^{2}+\left(\gamma_{u}\right)^{2}=1$. Many numerical tools can be used to rigorously solve for the reflection $\left(r_{p}\right)$ and transmission $\left(t_{u}\right)$ amplitudes by matching the tangential field components $H_{y}$ and $E_{x}$ at the metal-dielectric interface. ${ }^{12,13,18,19}$ Hereafter we use a frequency-domain modal method relying on Fourier-expansion techniques. The Fourierexpansion method ${ }^{20}$ is a generalization of the rigorous coupled-wave analysis ${ }^{21-24}$ initially used for grating analysis for arbitrary nonperiodic structures. In brief, the approach relies on an analytical integration of Maxwell's equations along the longitudinal $z$ axis and on a supercell approach in the $x$ direction. Perfectly matched layers ${ }^{25}$ implemented as nonlinear coordinate transforms ${ }^{26}$ are used in this direction to satisfy the outgoing wave conditions at the supercell boundaries. Since these layers absorb nonevanescent radiations like the generated SPP modes, the electromagnetic fields are null on the boundaries of the supercell and are thus periodic functions of the transversal coordinates. This allows the calculation of the radiated and guided modes in a Fourier (plane-wave) basis inside and below the slit. By matching the tangential field components at the metal-dielectric interface, the electromagnetic fields are calculated everywhere. Figures 2(a)-2(d) show the electromagnetic fields $H_{y}(x, z)$ and $E_{z}(x, z)$, which will be of main concern in the following for two different wavelengths, $\lambda=1.5$ and $0.6 \mu \mathrm{m}$, respectively. Because a saturated scale is used to reinforce the field in the vicinity of the slit, the standing-wave pattern in the slit does not show up. However, this scaling allows for the clear visualization of the near-field pattern, which exhibits a marked expansion of several wavelengths away from the slit aperture in medium 2. This is consistent with the involvement of SPPs at the slit aperture, but how do we recognize a quantitative signature of the SPPmode excitations in these near-field patterns?
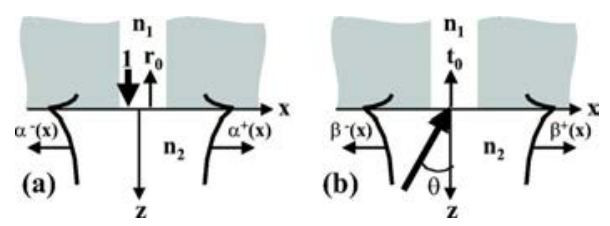

Fig. 1. SPP excitations at a metallic interface perforated by a single slit under illumination by (a) the fundamental mode of the slit or (b) a plane wave with an incidence angle $\theta$. The slit width is denoted by $w$, and $n_{1}$ and $n_{2}$ refer to the refractive indices inside and below the slit. $\alpha^{+}(x), \alpha^{-}(x), \beta^{+}(x)$, and $\beta^{-}(x)$ are the SPP generation coefficients defined for an incident wave with a unit power flow over the slit aperture area.
To clarify this question, we have developed an original approach that mainly exploits the completeness theorem for the normal modes of optical waveguides. ${ }^{27}$ This theorem has diverse applications in integrated optics, ranging from the proof of mode orthogonality to the formulation of coupled-mode equations, and provides a useful electromagnetic representation of light propagation in translationally invariant systems. It stipulates that any transverse field pattern of such a system can be decomposed in a linear combination of forward- and backward-traveling bounded and radiative modes. For our slit geometry and for $w / 2<x$ and $x<-w / 2$, it results that the transverse electromagnetic fields shown in Figs. 2(a)-2(d) can be expanded into the set of normal modes of a flat golddielectric interface:

$$
\begin{aligned}
& H_{y}=\left(\alpha^{+}(x)+\alpha^{-}(x)\right) H_{\mathrm{SP}}(z)+\Sigma_{\sigma} a_{\sigma}(x) H_{\sigma}^{(\mathrm{rad})}(z), \\
& E_{z}=\left(\alpha^{+}(x)-\alpha^{-}(x)\right) E_{\mathrm{SP}}(z)+\Sigma_{\sigma} a_{\sigma}(x) E_{\sigma}^{(\mathrm{rad})}(z) .
\end{aligned}
$$

In Eqs. (3a) and (3b), the transverse magnetic and electric fields $\left\{H_{\mathrm{SP}}, E_{\mathrm{SP}}\right\}$ of the bounded SPP mode is the analog of the guided mode of the waveguide theory, while the summation represents a continuum of radiation modes of the flat metal-dielectric interface. The SPP fields are know analytically $^{28}$ :

$$
H_{\mathrm{SP}}(z)=\left(N_{\mathrm{SP}}\right)^{-1} \exp \left(i \gamma_{\mathrm{SP}} z\right),
$$

with $N_{\mathrm{SP}}$ a normalization constant such that the SPP energy flow is unity and $\gamma_{\mathrm{SP}}=\left[\epsilon k^{2}-\left(k_{\mathrm{SP}}\right)^{2}\right]^{1 / 2}$ in the metal and $\left[\left(n_{2} k\right)^{2}-\left(k_{\mathrm{SP}}\right)^{2}\right]^{1 / 2}$ in the dielectric, respectively. $k_{\mathrm{SP}}$ is the well-known SPP propagation constant along the metal-dielectric interface and is given $\mathrm{by}^{28}$

$$
k_{\mathrm{SP}}=k \sqrt{\epsilon n_{2}^{2} /\left(\epsilon+n_{2}^{2}\right)} .
$$

In Eqs. (3a) and (3b) $\alpha^{+}(x)$ and $\alpha^{-}(x)$ are important coefficients that play a central role related to the SPP generation. The plus and minus superscripts refer to SPP propagating forward and backward. The $x$ dependence is known analytically: $\quad \alpha^{+}(x)=\alpha^{+}(w / 2) \exp \left(i k_{\mathrm{SP}}(x-w / 2)\right)$ and $\alpha(x)$ $=\alpha(w / 2) \exp \left(-i k_{\mathrm{SP}}(x+w / 2)\right)$, where $\alpha^{+}(w / 2)$ and $\alpha^{-}($ $-w / 2)$ represent the complex unknown coefficients related to the strength of the SPP excitation at the exit sides of the slit $(x= \pm w / 2)$. Using the mode orthogonality condition, ${ }^{26}$ we obtain for $\alpha^{+}$and $\alpha^{-}$

$$
\begin{aligned}
& \int_{-\infty}^{\infty} \mathrm{d} z H_{y}(x, z) E_{\mathrm{SP}}(z)=2\left(\alpha^{+}(x)+\alpha^{-}(x)\right), \\
& \int_{-\infty}^{\infty} \mathrm{d} z E_{z}(x, z) H_{\mathrm{SP}}(z)=2\left(\alpha^{+}(x)-\alpha^{-}(x)\right) .
\end{aligned}
$$

Because we are concerned by lossy metals with a finite conductivity, the modes are not orthogonal in the sense of the power flow as is usually the case in waveguide theory with weakly absorbing materials. This is the reason why we have used the unconjugate general form of orthogonality $^{29}$ in Eqs. (6a) and (6b) with $E H$ products instead of the $E H^{*}$ product often used in waveguide theory. The coefficients $\alpha^{+}(x)$ and $\alpha^{-}(x)$ are obtained by calculating numerically the overlap integrals on the left-hand 

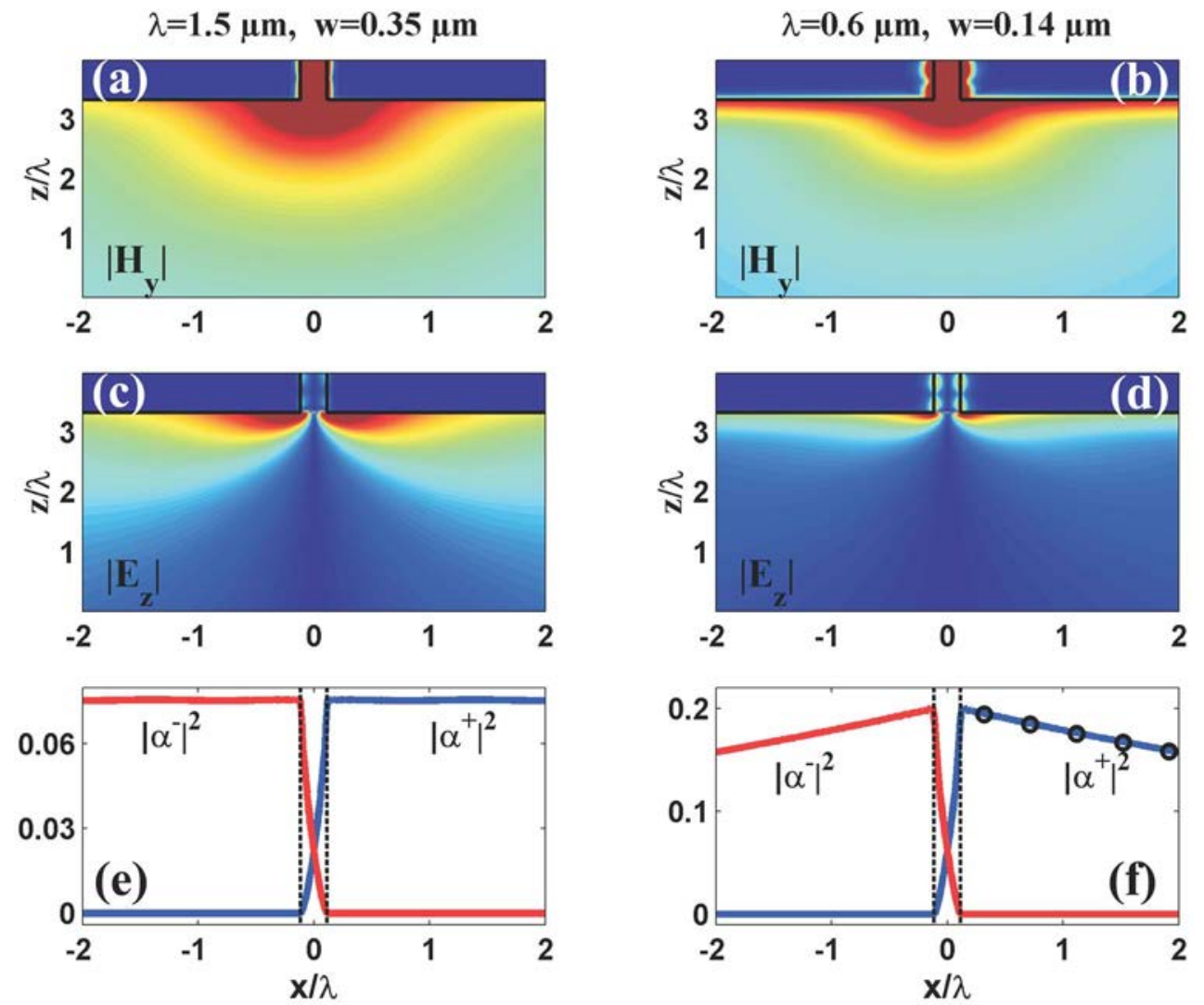

Fig. 2. (Color online) Validation of the rigorous formalism for the calculation of the SPP coupling coefficients. (a)-(d) Near-field patterns generated at a gold interface under illumination by the fundamental slit mode. (a) and (b) $\left|H_{y}\right|$; (c) and (d) $\left|E_{z}\right|$. Red is high magnitude, blue is low, and a saturated scale is used to reinforce the field in the vicinity of the slit. (e) and (f) SPP generation strengths $\left|\alpha^{+}(x)\right|^{2}$ and $\left|\alpha^{-}(x)\right|^{2}$ obtained from the near-field patterns shown in (a)-(d) by calculating the overlap integrals of Eqs. (6a) and (6b). In (f), the circles are numerical data equal to $\left|\alpha^{+}(w / 2)\right|^{2} \exp \left[-2 \operatorname{Im}\left(k_{\mathrm{SP}}\right)(x-w / 2)\right]$ with $\left|\alpha^{+}(w / 2)\right|^{2}=0.202$. (a), (c), and (e) are obtained for $\lambda=1.5 \mu \mathrm{m}$ and $w=0.35 \mu \mathrm{m}$; (b), (d), and (f) are for $\lambda=0.6 \mu \mathrm{m}$ and $w=0.14 \mu \mathrm{m}$. Other parameters are $n_{1}=n_{2}=1$.

sides of Eqs. (6a) and (6b). Figures 2(e) and 2(f) represent the modulus squared of these coefficients for the scattering problems considered in Figs. 2(a)-2(d). The results are obtained for an incident slit mode with a unit intensity; thus $\left|\alpha^{+}(x)\right|^{2}$ and $\left|\alpha^{-}(x)\right|^{2}$ represent the normalized SPP excitation strength. For $-w / 2<x<w / 2$, the coefficients are meaningless since there is no air-metal interface. Let us consider $\left|\alpha^{+}(x)\right|^{2}$; the discussion is similar for $\left|\alpha^{-}(x)\right|^{2}$. For $x>w / 2$, the computed $\left|\alpha^{+}(x)\right|^{2}$ values perfectly fulfill the expected SPP attenuation law $\left|\alpha^{+}(w / 2)\right|^{2} \exp \left[-2 \operatorname{Im}\left(k_{\mathrm{SP}}\right)(x-w / 2)\right]$, which are shown as circles in Fig. 2(f). For $\lambda=1.5 \mu \mathrm{m},\left|\alpha^{+}(x)\right|^{2}$ is found to be nearly independent of $x$ in the $2 \lambda$ large $x$ scale used in Fig. $2(\mathrm{e})$. This is again consistent with the SPP attenuation law since the 1/e SPP attenuation length at $\lambda=1.5 \mu \mathrm{m}$ for gold is $130 \lambda$. Further analysis, not reported here, has shown that the phase dependence of $\alpha^{+}(x)$ exactly coincides with the SPP propagation constant $\exp \left[-i \operatorname{Re}\left(k_{\mathrm{SP}}\right)\right.$ $\times(x-w / 2)]$. In addition, let us note that, consistent with the outgoing radiation condition at the slit output, $\left|\alpha^{+}(x)\right|^{2}$ (resp. $\left.\left|\alpha^{-}(x)\right|^{2}\right) \mid$ is found to be approximately null $\left(<10^{-6}\right)$ for $x<w / 2$ (resp. $x>w / 2)$.

Figure 3 shows the variation of the main relevant quantities involved in the scattering problem as a function of the slit width. All results are obtained for an incident slit mode with a unitary power. The modal reflectiv-
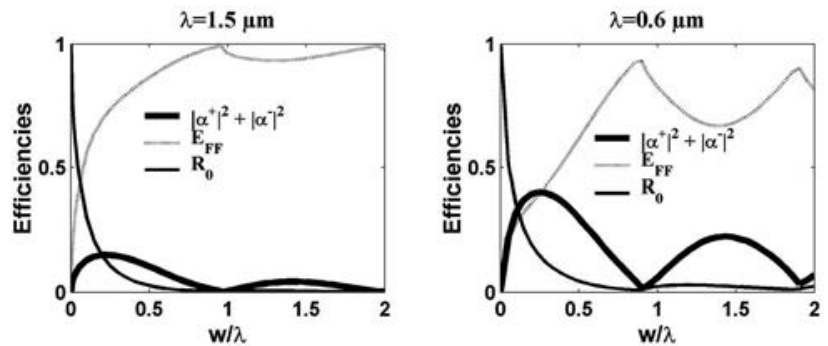

Fig. 3. Variation of the main physical quantities associated with the scattering problems considered in Fig. 2 as a function of the slit width. $\left|\alpha^{+}(x)\right|^{2}+\left|\alpha^{-}(x)\right|^{2}$ represents the total SPP excitation, $R_{0}=\left|r_{0}\right|^{2}$ is the modal reflectivity, and $E_{\mathrm{FF}}$ is the far-field energy radiated in medium 2 . The power of the incident slit mode is 1 .

ity $R_{0}=\left|r_{0}\right|^{2}$, shown with a thin solid curve, monotonously decreases with $w$ and reaches unity in the limit of $w \rightarrow 0$. The total power $E_{\mathrm{FF}}$ radiated into the far field in medium 2 exhibits a more intricate behavior (see the gray curve). It strongly depends on the wavelength, i.e., on the dielectric properties of the metal, and is maximum whenever $w$ is approximately a multiple of the wavelength that corresponds to the passing off of symmetric evanescent slit modes, which become propagative. This intricate behavior reflects that of the total SPP excitation efficiency, $\left|\alpha^{+}(w / 2)\right|^{2}+\left|\alpha^{-}(-w / 2)\right|^{2}$, which will be discussed in more detail in Section 3 through a semianalytical model. 


\section{APPROXIMATE MODEL}

To obtain useful and analytical expressions for the SPP generation without relying on a fully vectorial software to solve Maxwell's equations, we have developed an intuitive approximate model. As suggested by the previous analysis, the SPP generation results from a two-step mechanism: a purely geometric diffraction problem followed by the launching of the bounded SPP modes on the flat interfaces surrounding the slit. An analytic treatment has to rely on assumptions. We assume that the diffraction problem that results in a specific nearfield distribution in the immediate vicinity of the slit aperture [the $H_{y}, E_{z}$ fields of Eqs. (6a) and (6b) at $x= \pm w / 2$ ] is weakly dependent on the dielectric properties of the metal and that it can be estimated by considering the metal as a perfect conductor (PC). On the contrary, the launching of the bounded SSP mode strongly depends on the intrinsic dielectric properties of the metal-dielectric interface [the $H_{\mathrm{SP}}, E_{\mathrm{SP}}$ fields of Eqs. (6a) and (6b)]. In other words, the model takes into account the main physical properties of the two-step mechanism carefully and independently. Assuming that the metal is perfectly conducting for solving the geometrical scattering drastically reduces the complexity. We have further assumed that the field inside the slit is composed of the forward- and backward-reflected fundamental mode. The approach developed below for calculating the near-field distribution obtained for a slit in a perfect metal largely follows that developed in Ref. 30 for light diffraction by slit arrays.

\section{A. Slit-Mode Illumination}

Under the one-mode approximation, Eq. (1) becomes

$$
H_{1}(x, z)=\left(1+r_{0}\right) \Psi_{0}(x, z),
$$

where the fundamental mode $\Psi_{0}(x, z)$ is given by $\Psi_{0}(x, z)=\left(N_{0}\right)^{-1 / 2} \operatorname{rect}(x / w) \exp \left(-i k n_{1} z\right)$, with $N_{0}$ $=w /\left(2 \epsilon_{0} n_{1}\right)$ a normalization constant such that the power flow is unitary. The function $\operatorname{rect}(x)$ is defined by $\operatorname{rect}(x)$ $=1$ if $|x|<1 / 2$, and 0 otherwise. Equation (2) is unchanged. By matching the PC boundary conditions at $z$ $=0$ (continuity of $E_{x}$ for any $x$ and continuity of $H_{y}$ over the slit apertures), one easily obtains for the field radiated in medium 2

$$
t_{u}=\left(N_{0}\right)^{-1 / 2} \frac{2\left(n_{2} / n_{1}\right) w^{\prime}}{\left(1+\left(n_{2} / n_{1}\right) w^{\prime} I_{0}\right)} \frac{\operatorname{sinc}\left(\pi w^{\prime} u\right)}{\gamma_{u}},
$$

where $w^{\prime}=w /\left(\lambda / n_{2}\right)$ is the normalized slit width, $I_{0}$ $=\int_{-\infty}^{\infty} \mathrm{d} u \operatorname{sinc}^{2}\left(\pi w^{\prime} u\right) / \gamma_{u}$, and $\operatorname{sinc}(\cdot)=\sin (\cdot) /(\cdot)$. Similarly, the modal reflectivity coefficient $r_{0}$ defining the field in medium 1 is shown to be given by

$$
r_{0}=\frac{\left(n_{2} / n_{1}\right) w^{\prime} I_{0}-1}{\left(n_{2} / n_{1}\right) w^{\prime} I_{0}+1} .
$$

Equations (7), (2), (8), and (9) completely determine the electromagnetic fields scattered in media 1 and 2. From this near-field distribution, the overlap integrals of Eqs. (6a) and (6b) are now considered. These integrals that hold for $|x|>w / 2$ are no longer valid for the perfectly conducting case, but according to our ansatz, remains approximately valid in the close vicinity of the slit aperture.
By writing Eqs. (6a) and (6b) for $x= \pm w / 2$ and then by exploiting the mirror symmetry for $H_{y}$ and $E_{z}$ with respect to the plane $x=0$, one obtains

$$
2 \alpha^{+}(w / 2)=2 \alpha^{-}(-w / 2)=-\int_{0}^{\infty} \mathrm{d} z H_{y}(x, w / 2) E_{\mathrm{SP}}(z),
$$

where the integrand runs from 0 to $+\infty$ since the field in the metal is equal to zero under the perfectly conducting condition used in the model. To derive Eq. (10), we have additionally assumed that $\alpha^{+}(-w / 2)=\alpha^{-}(w / 2)=0$; this is reasonable according to Section 2. From Eqs. (2), (8), and (10) and for $N_{\mathrm{SP}} \approx|\epsilon|^{1 / 2} /\left(4 \omega \epsilon_{0} n_{2}^{3}\right)$, one gets for the SSP generation strength $\alpha$ on both sides of the aperture

$$
\begin{aligned}
\alpha & =\alpha^{+}(w / 2)=\alpha^{-}(-w / 2) \\
& =-i\left(\frac{4}{\pi} \frac{n_{2}^{2}}{\pi n_{1}} \frac{\sqrt{|\epsilon|}}{\left(-\epsilon-n_{2}^{2}\right)} w^{\prime}\right)^{1 / 2} \frac{I_{1}}{1+\left(n_{2} / n_{1}\right) w^{\prime} I_{0}},
\end{aligned}
$$

with

$$
I_{1}=\int_{-\infty}^{\infty} \mathrm{d} u \frac{\operatorname{sinc}\left(\pi w^{\prime} u\right) \exp \left(-i \pi w^{\prime} u\right)}{\gamma_{u}\left(\gamma_{u}+\sqrt{n_{2}^{2} /\left(\epsilon+n_{2}^{2}\right)}\right)} .
$$

The corresponding efficiencies $|\alpha|^{2}$ are given by

$$
\begin{aligned}
|\alpha|^{2} & =\left|\alpha^{+}(w / 2)\right|^{2}=\left|\alpha^{-}(-w / 2)\right|^{2} \\
& =\frac{4 w^{\prime} n_{2}^{2}}{\pi n_{1}}\left|\frac{\epsilon^{1 / 2}}{\epsilon+n_{2}^{2}}\right|\left|\frac{I_{1}}{1+\left(n_{2} / n_{1}\right) w^{\prime} I_{0}}\right|^{2} .
\end{aligned}
$$

Equations (11) and (12) represent the semianalytical expressions for the complex SPP excitation coefficients and the SPP excitation efficiencies when the slit aperture is illuminated by the fundamental slit mode. The integrals $I_{1}$ and $I_{0}$ are calculated numerically.

\section{B. Plane-Wave Illumination}

Let us now consider the scattering problem shown in Fig. 1(b). Inside the slit, the magnetic field $H_{1}$ admits a modal expansion of the form

$$
H_{1}(x, z)=t_{0} \Psi_{0}(x, z)
$$

where $t_{0}$ is the unknown modal transmission coefficient. In medium 2 below the slit, the magnetic field $H_{2}$ can be expanded into a plane-wave basis:

$$
\begin{aligned}
H_{2}(x, z)= & \left(N_{P}\right)^{-1 / 2} \exp \left[i k n_{2} \sin (\theta) x-i k n_{2} \cos (\theta) z\right] \\
& +\int_{-\infty}^{\infty} \mathrm{d} u r_{u} \exp \left(i k n_{2} u x\right) \exp \left(i k n_{2} \gamma_{u} z\right),
\end{aligned}
$$

where $r_{u}$ are unknown plane-wave reflection coefficients and $N_{P}=(w \cos (\theta)) /\left(2 \epsilon_{0} n_{2}\right)$ is a normalization constant such that the power flow of the incident plane wave on the slit aperture is unitary. By matching the PC boundary conditions at $z=0$, one obtains

$$
t_{0}=\left(N_{0} / N_{P}\right)^{1 / 2} \frac{2 \operatorname{sinc}\left(\pi w^{\prime} \sin (\theta)\right)}{\left(n_{2} / n_{1}\right) w^{\prime} I_{0}+1}
$$

for the modal transmission and 


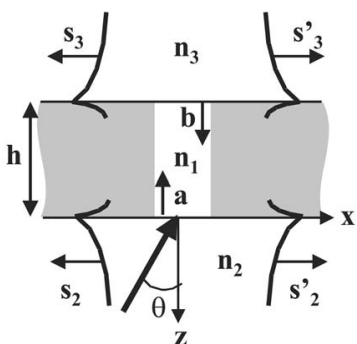

Fig. 4. SPP excitations at a slit perforated in a metal film (thickness $h$ ) sandwiched between two uniform media of refractive indices $n_{2}$ and $n_{3}$ and illuminated by a plane wave with an incidence angle $\theta$. Inside the slit the refractive index is denoted by $n_{1}$. The $s_{2}, s_{2}^{\prime}, s_{3}, s_{3}^{\prime}$ represent the SPP generation coefficients at the top and bottom interfaces.

$$
r_{u}=\delta(\theta)-t_{0}\left(N_{0} / N_{P}\right)^{1 / 2} w^{\prime} \frac{n_{2}}{n_{1}} \frac{\operatorname{sinc}\left(\pi w^{\prime} u\right)}{\left(1-u^{2}\right)^{1 / 2}}
$$

for the plane-wave expansion coefficients. In Eq. (16), $\delta$ represents the Dirac distribution. Using the same approach as that used in subsection 3.A, we obtain

$$
\begin{aligned}
\beta & =\beta^{+}(w / 2)=\beta^{-}(-w / 2) \\
& =-\left(N_{0} / N_{p}\right)^{1 / 2} \sin c\left[\pi w^{\prime} \sin (\theta)\right] \alpha^{+}(w / 2),
\end{aligned}
$$

where $\beta^{+}(w / 2)$ and $\beta^{-}(-w / 2)$ denote the SPP generation coefficients for the plane-wave illumination case. The corresponding efficiencies are given by

$$
\begin{aligned}
|\beta|^{2} & =\left|\beta^{+}(w / 2)\right|^{2}=\left|\beta^{-}(-w / 2)\right|^{2} \\
& =\frac{4 w^{\prime} n_{2}^{3}}{\pi \cos (\theta) n_{1}^{2}}\left|\frac{\epsilon^{1 / 2}}{\epsilon+n_{2}^{2}}\right|\left|\frac{I_{1} \operatorname{sinc}\left(\pi w^{\prime} \sin (\theta)\right)}{1+\left(n_{2} / n_{1}\right) w^{\prime} I_{0}}\right|^{2} .
\end{aligned}
$$

\section{Surface-Plasmon Polariton Generation at Slits and Grooves in a Metal Film}

The SPP generation coefficients $\alpha$ and $\beta$ represent basic scattering quantities that may be used to analyze more sophisticated diffraction situations. For instance, let us consider the scattering problem by a slit perforated in a metal film deposited on a substrate with a refractive index $n_{2}$ and illuminated by a plane wave incident from the substrate; see Fig. 4 for a definition of the parameters. Let us denote by $a$ and $b$ the unknown modal coefficients of the fundamental mode in the slit traveling upward and downward, respectively. If one neglects higher-order modes in the slits, ${ }^{31}$ the master coupled-mode equations for this system are easily derived using a Fabry-Perot model. With obvious notation one gets

$$
\begin{aligned}
& a=t_{21}+r_{12} b \exp \left(i k n_{0}^{\text {eff }} h\right), \\
& b=r_{13} a \exp \left(i k n_{0}^{\text {eff }} h\right),
\end{aligned}
$$

where $n_{0}^{\text {eff }}$ is the effective index of the fundamental mode in the slit, and $t_{21}, r_{12}$, and $r_{13}$ are slit-mode coupling coefficients related to the $r_{0}$ and $t_{0}$ coefficients defined in Eqs. (9) and (15). From Subsections 3.A and 3.B, the SPP generation coefficients $s_{2}, s_{2}^{\prime}, s_{3}, s_{3}^{\prime}$ are given by $s_{2}=s_{2}^{\prime}=\beta$ $+b \alpha \exp \left(i k n_{0}^{\text {eff }} h\right)$ and $s_{3}=s_{3}^{\prime}=a \alpha \exp \left(i k n_{0}^{\text {eff }} h\right)$. From those equations and after solving Eqs. (19a) and (19b) for $a$ and $b$, one obtains for the SPP generation coefficients at the slit interfaces

$$
\begin{aligned}
& s_{2}=s_{2}^{\prime}=\beta+\frac{t_{21} r_{13} \alpha \exp \left(2 i k n_{0}^{\mathrm{eff}} h\right)}{1-r_{12} r_{13} \exp \left(2 i k n_{0}^{\mathrm{eff}} h\right)}, \\
& s_{3}=s_{3}^{\prime}=\frac{t_{21} \alpha \exp \left(i k n_{0}^{\mathrm{eff}} h\right)}{1-r_{12} r_{13} \exp \left(2 i k n_{0}^{\mathrm{eff}} h\right)} .
\end{aligned}
$$

These equations show that the optimization of the SPP generation efficiency $s_{3}=s_{3}^{\prime}$ on the upper side solely consists in setting up a waveguide resonance in the slit by fulfilling the Fabry-Perot condition, i.e., by choosing the metallic film thickness so that $1-r_{12} r_{13} \exp \left(2 i k n_{0}^{\text {eff }} h\right)$ is minimal. On the illuminated side, the SPP generation efficiency $s_{2}=s_{2}^{\prime}$ takes a more complicated form, and its optimization additionally requires a phase matching between the two terms on the right side of Eq. (20a).

\section{MODEL PREDICTIONS, VALIDATIONS, AND LIMITATIONS}

\section{A. Model Predictions}

The solid curves in Figs. 5 and 6 shows the SPP excitation efficiencies predicted by the approximate model for the two scattering geometries of Fig. 1 and for a broad range of wavelengths from the visible to the thermal infrared. The integrals $I_{0}$ and $I_{1}$ in Eqs. (12) and (18) are calculated numerically. ${ }^{32}$ Table 1 provides numerical data of $I_{0}$ and $I_{1}$ for several values of $w^{\prime}$ and for $\epsilon=-26.27+1.85 i$ (gold at $\lambda=800 \mathrm{~nm}$ ). Note that $I_{0}$ depends on a single parameter $w^{\prime}$, whereas $I_{1}$ depends on the metal permittivity and on $w^{\prime}$. All plots in Figs. 5 and 6 are relative to the total SPP excitation efficiency $e_{\mathrm{SP}}$ on both sides of the aperture, $e_{\mathrm{SP}}$ being equal to $\left|\alpha^{+}(w / 2)\right|^{2}+\left|\alpha^{-}(-w / 2)\right|^{2}$ in Fig. 5 and to $\left|\beta^{+}(w / 2)\right|^{2}+\left|\beta^{-}(-w / 2)\right|^{2}$ in Fig. 6. They are obtained for an (a)

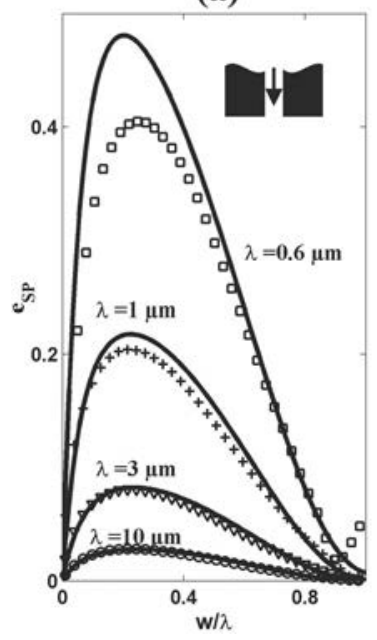

(b)

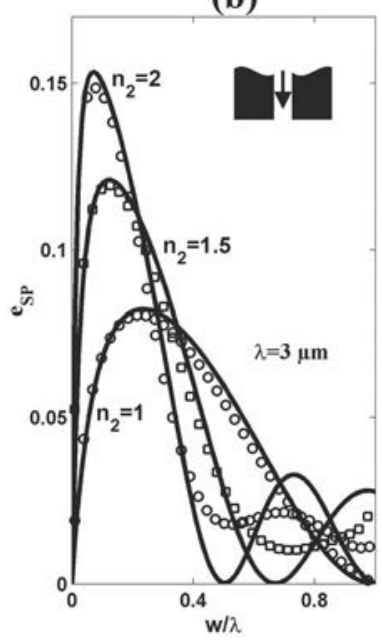

Fig. 5. Total SPP generation efficiencies $e_{\mathrm{SP}}=\left|\alpha^{+}(x)\right|^{2}+\left|\alpha^{-}(x)\right|^{2}$ for a slit illuminated by its fundamental guided mode as a function of the slit width. Solid curves represent the model predictions. The symbols represent the calculated data obtained with the rigorous formalism. (a) Influence of the metal (gold) permittivity, $n_{1}=n_{2}=1$. (b) Influence of the substrate refractive index $n_{2}, n_{1}$ $=1$ and $\lambda=3 \mu \mathrm{m}$. 
(a)

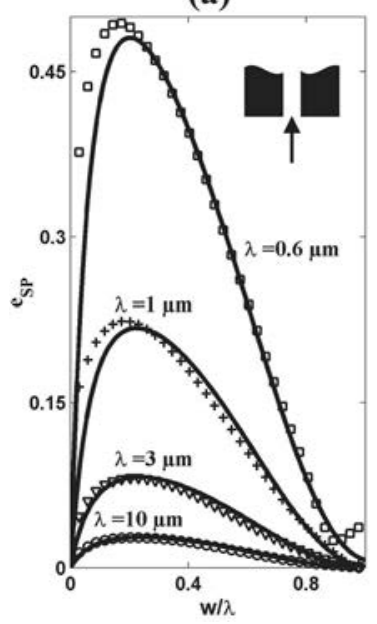

(b)

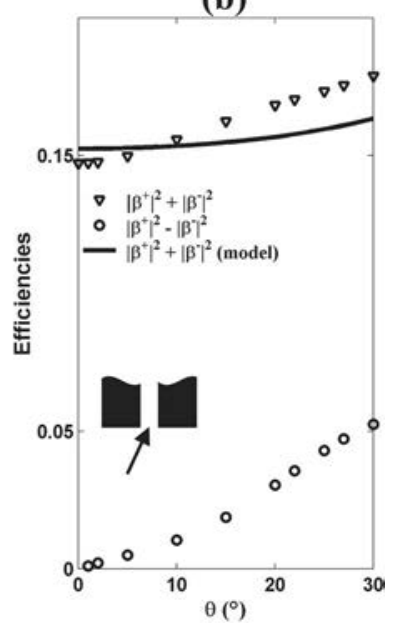

Fig. 6. SPP generation efficiencies for a slit illuminated by a plane wave, (a) $e_{\mathrm{SP}}=\left|\beta^{+}(w / 2)\right|^{2}+\left|\beta^{-}(-w / 2)\right|^{2}$ as a function of the slit width for different wavelengths, $\lambda=0.6,1,3$, and $10 \mu \mathrm{m}$, for $n_{1}=n_{2}=1$ and $\theta=0$. (b) Influence of the incident angle for $\lambda$ $=1.5 \mu \mathrm{m}$; other parameters are $n_{1}=n_{2}=1$ and $w / \lambda=0.3$. The model does not predict any difference between $\left|\beta^{+}(w / 2)\right|^{2}$ and $\left|\beta^{-}(-w / 2)\right|^{2}$ for any $\theta$. In (a) and (b), the solid curves represent the model predictions and the symbols represent the calculated data obtained with the rigorous formalism.

Table 1. $I_{1}$ and $I_{0}$ for Gold at $\lambda=800 \mathrm{~nm}$ $(\epsilon=-26.27+1.85 i)$

\begin{tabular}{ccccc}
\hline$w^{\prime}$ & 0.1 & 0.3 & 0.5 & 0.7 \\
$I_{0}$ & $3.09-4.09 i$ & $2.72-1.68 i$ & $2.13-0.63 i$ & $1.54-0.18 i$ \\
$I_{1}$ & $0.53-2.93 i$ & $1.75-1.80 i$ & $1.79-0.40 i$ & $1.01+0.35 i$ \\
\hline
\end{tabular}

incident light with a unit power per slit opening area. Figures 5(a) and 6(a) show the SPP excitation efficiencies for illuminations by the slit mode and by the plane wave, respectively. These efficiencies strongly depend on the slit width and on the metal-dielectric properties. They are maximum for an optimal slit width of $w \approx 0.23 \lambda$, a value nearly independent of the wavelength of interest. At visible wavelength, $\lambda=0.6 \mu \mathrm{m}$, the efficiency is fairly large and reaches a value as high as $\approx 0.45$ for the optimal slit width; as much light is scattered into the SPPs as it is radiated in the far field. This result is not specific to the gold metal used in the example. Other noble metals used at frequencies close to the plasma frequency exhibit similar behavior, as shown by the $\epsilon^{1 / 2} /\left(\epsilon+\left(n_{2}\right)^{2}\right)$ dependence in the expressions of Eqs. (12) and (18). For large wavelengths, the efficiency rapidly decreases; it is only $2.8 \%$ for $\lambda=10 \mu \mathrm{m}$. From Eqs. (12) and (18), it is easily shown that the efficiency scales as $|\epsilon(\lambda)|^{-1 / 2}$ since $\left(n_{2}\right)^{2} \ll|\epsilon|$. Figure 5(b) shows the effect of the substrate refractive index when the slit aperture is illuminated by the fundamental slit mode. The predictions are obtained for $\lambda=3 \mu \mathrm{m}$ and $n_{1}=1$. As $n_{2}$ increases, narrower slits provide better SPP excitations and the slit width $w_{0}=\lambda / n_{2}$, which corresponds to a null excitation shift toward smaller values of $w$. This is a direct consequence of the $w^{\prime}=n_{2} w / \lambda$ dependence of the $I_{0}$ and $I_{1}$ integrals in Eqs. (12) and (18). Additionally we note that the peak value of the SPP excitation increases with $n_{2}\left(\propto n_{2} / n_{1}\right)$, and that the optimal slit width slightly decreases. Figure 6(b) shows the influence of the angle of incidence on the SPP excitation for $\lambda$ $=1.5 \mu \mathrm{m}$ and $w / \lambda=0.3$. A slight increase of the total excitation $e_{\mathrm{SP}}$ with $\theta$ is predicted. Note that the model additionally predicts that $\left|\beta^{+}(w / 2)\right|^{2}=\left|\beta^{-}(-w / 2)\right|^{2}$ for any $\theta$ values, see Eq. (18). This questionable result will be discussed below.

\section{B. Model Validation}

To validate the model predictions, we have provided extensive computational calculations for the SPP excitation, strictly following the procedure described in Section 2 . The results of the theoretical formalism are shown by the symbols in Figs. 5 and 6. Overall, the exact data quantitatively agree with the model predictions and confirm the general trends discussed previously, like the existence of an optimal slit width, the influence of the angle of incidence, and of the metal-dielectric properties. We first note that the larger the wavelength, the better the agreement, consistent with the PC metal approximation used in the model. Some discrepancies between the model predictions and the calculated data are also observed. As shown in Fig. 5(b), the null excitation predicted for $w_{0}$ $=\lambda / n_{2}$ by the model is not observed for $n_{2} \neq 1$. Additionally, the calculated data show a monotonous increase of $\left|\beta^{+}(w / 2)\right|^{2}-\left|\beta^{-}(-w / 2)\right|^{2}$ with $\theta$, see the circles in Fig. 6(b).

In Fig. 7 we provide a comparison between the model predictions (solid curves) and data obtained with the rigorous formalism (squares) for SPP generation strengths at a groove perforated in a metal substrate. This configuration is interesting since groovelike scratches are often used in practice to excite SPP waves for device characterizations. Plots are shown as a function of the groove depth $h$ for $\lambda=0.8 \mu \mathrm{m}$ and $w / \lambda=0.1$ in Fig. 7 (a) and for $\lambda$ $=1.5 \mu \mathrm{m}$ and $w / \lambda=0.1$ in Fig. $7(\mathrm{~b})$. The model predictions are obtained with Eq. (20a) for $n_{1}=n_{2}=1$ and $n_{3}=\epsilon^{1 / 2}$; the effective index $n_{0}^{\text {eff }}$ is calculated using the Fourier modal method described in Ref. 20. We further assume that the
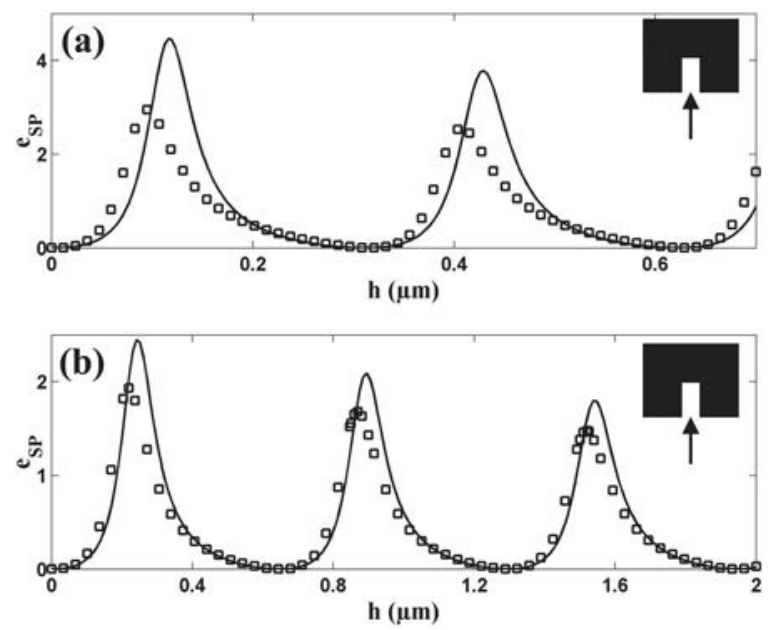

Fig. 7. Total SPP generation efficiency at a groove perforated in a gold substrate as a function of the groove depth $h$ for normally incident light $(\theta=0)$. Solid curves, model predictions obtained with Eq. (20a) for $n_{1}=n_{2}=1$ and $n_{3}=\epsilon^{1 / 2}$. Squares, calculated data using the rigorous formalism and the Fourier modal method. (a) $\lambda=0.8 \mu \mathrm{m}, w / \lambda=0.1$, and $n_{0}^{\text {eff }}=1.29+0.0098 i$. (b) $\lambda=1.5 \mu \mathrm{m}, w / \lambda$ $=0.1$, and $n_{0}^{\text {eff }}=1.16+0.0084 i$. 

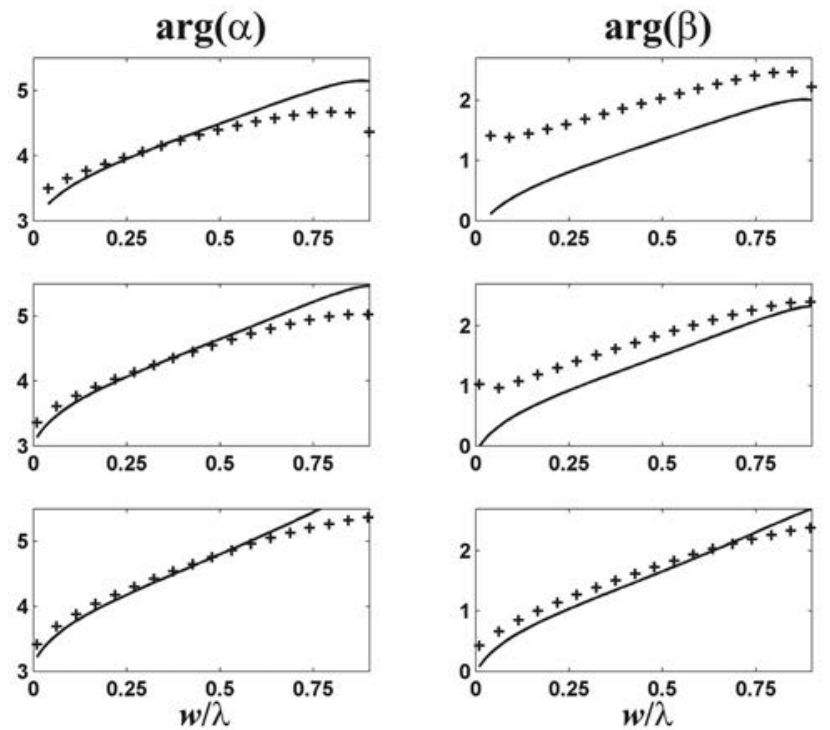

Fig. 8. Phase factors associated with the SPP scattering processes. Solid curve, model predictions obtained with Eqs. (11) and (17). Plusses, computational data obtained with the rigorous formalism. From top to bottom, $\lambda=0.6,0.8$, and $3 \mu \mathrm{m}$.

modal reflectivity $r_{13}$ of the fundamental slit mode at the metal-groove interface is equal to one. The model quantitatively agrees with the calculated data and well predicts the most interesting aspect, namely, the SPP generation enhancement obtained for Fabry-Perot slit resonances. At these resonances, the light generated into the two SPP waves is two to three times larger than that which is directly incident onto the aperture area. We additionally note that the excitation maxima are slightly overestimated. This can be attributed to the fact that the model overestimates the SPP generation efficiencies $\left|\alpha^{+}(w / 2)\right|^{2}$ and $\left|\alpha^{-}(-w / 2)\right|^{2}$ [see Fig. 5(a)] and to the unitary assumption for $r_{13}$. The resonance predicted by the model is also shown to be shifted slightly toward large depth values. This can be understood by considering that, within the model, the phase of the $r_{12}$ and $r_{13}$ coefficients are only approximately predicted. For narrow grooves like those considered in Fig. 7, skin depth effects cannot be neglected, and the phase delays for the modal reflectivity depart from those predicted with a PC metal approximation.

So far, we have discussed only SPP generation efficiencies. To further validate the model, we now focus on the phase factor $\arg (\alpha)$ and $\arg (\beta)$ associated with the SPP scattering processes as described by Eqs. (11) and (17). Figure 8 shows a comparison between the model predictions (solid curves) and the computational data obtained with the rigorous formalism (plusses) for $\lambda=0.6,0.8$, and $3 \mu \mathrm{m}$. The model captures the general trend, i.e., the increase of the phase factor with the slit width. As we previously noted for the SPP generation strengths, the model is more accurate for $\alpha$ than for $\beta$ and for large wavelengths.

\section{Model Limitations}

The inaccuracies of the model predictions are due to the two approximations used to derive the near-field pattern in the vicinity of the slit aperture: the perfectly conducting approximation and the one-mode model used to de- scribe the field in the slit [see Eqs. (7) and (13)]. The perfectly conducting assumption contributes only weakly to inaccuracies. Although they are all the more accurate as the wavelength is large [see Figs. 5(a) and 6(a)], the model predictions remain rather accurate even if the metal permittivity is low, as is the case for gold at $\lambda$ $=0.6 \mu \mathrm{m}$. The more severe limitation results from the onemode approximation. This approximation does not hold when several nonevanescent modes exist in the slit. The cutoff of the second (antisymmetric) mode is $w / \lambda=0.5 n_{1}$ and for the third (symmetric) mode is $w / \lambda=n_{1}$ under the ideal metal approximation. Because of symmetry reasons, the second mode is not excited when the slit aperture is illuminated by the fundamental guided mode or by the plane wave under normal incidence. This is the reason why the model remains accurate even for large slit widths up to $w / \lambda=1$ in Figs. 5(a) and 6(a). For $w / \lambda>1$, a much larger discrepancy is observed, especially for small wavelengths in the visible part of the spectrum. Another important consequence of the one-mode approximation is the prediction of identical excitation efficiencies $\left|\beta^{+}(w / 2)\right|^{2}$ and $\left|\beta^{-}(-w / 2)\right|^{2}$ for a plane-wave illumination and for $\theta$ $\neq 0$ [see Eq. (18)]. This is a direct consequence of the symmetry imposed by the one-mode approximation in the slit, which reflects in the plane-wave expansion of the scattered field [Eq. (14)] by matching the boundary conditions. As shown in Fig. 6(b), oblique illumination indeed results in a difference in the SPP excitation efficiencies on the right and left sides of the slit aperture. Although we have not performed a systematic rigorous study, we have noted as a general trend that the difference increases as the slit width increases. Let us note that although it cannot predict any difference, the model accurately predicts the total SPP excitation $\left|\beta^{+}(w / 2)\right|^{2}+\left|\beta^{-}(-w / 2)\right|^{2}$ [see Fig. $6(\mathrm{~b})]$. Further improvements of the model may consist in using surface impedance boundary conditions ${ }^{33}$ for enhancing the accuracy at visible wavelength or in using a double-mode expansion in the slit. The latter leads again to analytical expressions for the SPP excitation efficiencies at the expense of additional complexity.

\section{CONCLUSION}

We have studied the generation of SPP waves at a metallic interface perforated by an isolated subwavelength slit. We have derived semianalytical expressions for the SPP scattering coefficients and efficiencies at the slit aperture. The model, which provides a microscopic description for SPP generation mechanisms at slit apertures, has been applied to the analysis of a variety of slit and groove geometries that are of interest in practice. It provides a comprehensive discussion of the influence of the main scattering problem parameters, like the normalized slit width, the angle of incidence, and the effect of the dielectric properties of the metallic and dielectric materials. An important outcome of the model is the prediction of a peak SPP excitation efficiency for an optimal subwavelength slit width of $w \approx 0.23 \lambda$. This peak efficiency is fairly large for metals with a low conductivity like gold in the visible regime; in this case, the fraction of the incident radiation that couples into the SPP reaches a value as high as the total far-field radiated energy. This indicates that even 
simple metallic structures, like slits, may be used to efficiently manipulate SPP visible waves at a nanoscale level.

The model has been validated by comparisons with computational data obtained with a theoretical formalism relying on a rigorous solution of Maxwell's equations. A quantitative agreement has been obtained for the various geometries analyzed in this work. The model limitations or inaccuracies have been outlined and explained. We have further evidenced that the phases of the SPP scattering mechanisms at the slit aperture are correctly handled within the approach.

\section{ACKNOWLEDGMENT}

This work is partly supported by the Network of Excellence on Micro Optics (NEMO).

Corresponding author P. Lalanne's e-mail address is philippe.lalanne@iota.u-psud.fr.

\section{REFERENCES AND NOTES}

1. T. Thio, K. M. Pellerin, R. A. Linke, T. W. Ebbesen, and H. J. Lezec, "Enhanced light transmission through a single subwavelength aperture," Opt. Lett. 26, 1972-1974 (2001).

2. F. I. Baida and D. Van Labeke, "Light transmission by subwavelength annular aperture arrays in metallic films," Opt. Commun. 209, 17-22 (2002).

3. K. J. K. Koerkamp, S. Enoch, F. B. Segerink, N. F. van Hulst, and L. Kuipers, "Strong influence of hole shape on extraordinary transmission through periodic arrays of subwavelength holes,” Phys. Rev. Lett. 92, 183901 (2004).

4. H. J. Lezec, A. Degiron, E. Devaux, R. A. Linke, L. MartinMoreno, F. J. Garcia-Vidal, and T. W. Ebbesen, "Beaming light from a subwavelength aperture," Science 297, 820-822 (2002).

5. H. F. Schouten, N. Kuzmin, G. Dubois, T. D. Visser, G. Gbur, P. F. A. Alkemade, H. Blok, G. W. 't Hooft, D. Lenstra, and E. R. Eliel, "Plasmon-assisted two-slit transmission: Young's experiment revisited," Phys. Rev. Lett. 94, 053901 (2005).

6. H. Bethe, "Theory of diffraction by small holes," Phys. Rev. 66, 163-182 (1944).

7. C. J. Bouwkamp, "Diffraction theory," Rep. Prog. Phys. 17, 35-100 (1954).

8. A. Roberts, "Electromagnetic theory of diffraction by a circular aperture in a thick, perfectly conducting screen," J. Opt. Soc. Am. A 4, 1970-1983 (1987).

9. R. Wannemacher, "Plasmon-supported transmission of light through nanometric holes in metallic thin films," Opt. Commun. 195, 107-118 (2001).

10. S. H. Chang, S. K. Gray, and G. C. Schatz, "Surfaceplasmon generation and light transmission by isolated nanoholes and nanohole arrays in thin metal films," Opt. Express 13, 3150-3165 (2005).

11. Y. Xie, A. R. Zakharian, J. V. Moloney, and M. Mansuripur, "Transmission of light through slit aperture in metallic films," Opt. Express 12, 6106-6121 (2004).

12. P. Lalanne, J. C. Rodier, and J. P. Hugonin, "Surface plasmons of metallic surfaces perforated by nanohole arrays,” J. Opt. A Pure Appl. Opt. 7, 422-426 (2005).

13. L. Yin, V. K. Vlasko-Vlasov, A. Rydh, J. Pearson, U. Welp, S.-H. Chang, S. K. Gray, G. C. Schatz, D. E. Brown, and C.
W. Kimball, "Surface plasmons at single nanoholes in Au films," Appl. Phys. Lett. 85, 467-469 (2004).

14. H. Ditlbacher, J. R. Krenn, N. Felidj, B. Lamprecht, G. Schider, M. Salerno, A. Leitner, and F. R. Aussenegg, "Fluorescence imaging of surface plasmon fields," Appl. Phys. Lett. 80, 404-406 (2002).

15. P. Lalanne, J. P. Hugonin, and J. C. Rodier, "Theory of surface plasmon generation at nanoslit apertures," Phys. Rev. Lett. 95, 263902 (2005).

16. Inaccuracies due to numerical truncation in the modal expansions are inevitable in numerics but they are kept at a low level. We estimate that the scattering coefficients are calculated with a relative accuracy better than $1 \%$.

17. E. D. Palik, Handbook of Optical Constants of Solids (Academic, 1985), Part II.

18. P. W. Wei, H. L. Chou, Y. R. Cheng, C. H. Wei, W. Fann, and J. O. Tegenfeldt, "Beaming effect of optical near-field in multiple metallic slits with nanometric linewidth and micrometer pitch," Opt. Commun. 253, 198-204 (2005).

19. Y. Xie, A. R. Zakharian, J. V. Moloney, and M. Mansuripur, "Transmission of light through slit apertures in metallic films," Opt. Express 12, 6106-6121 (2004).

20. E. Silberstein, P. Lalanne, J. P. Hugonin, and Q. Cao, "Use of grating theories in integrated optics," J. Opt. Soc. Am. A 18, 2865-2875 (2001).

21. M. G. Moharam, E. B. Grann, D. A. Pommet, and T. K. Gaylord, "Formulation for stable and efficient implementation of the rigorous coupled-wave analysis of binary gratings,” J. Opt. Soc. Am. A 12, 1068-1076 (1995).

22. Ph. Lalanne and G. M. Morris, "Highly improved convergence of the coupled-wave method for TM polarization," J. Opt. Soc. Am. A 13, 779-784 (1996).

23. G. Granet and B. Guizal, "Efficient implementation of the coupled-wave method for metallic lamellar gratings in TM polarization," J. Opt. Soc. Am. A 13, 1019-1023 (1996).

24. L. Li, "Mathematical reflections on the Fourier modal method in grating theory," in Mathematical Modeling in Optical Science, Frontiers in Applied Mathematics, G. Bao, L. Cowsar, and W. Masters, eds. (Society for Industrial and Applied Mathematics, 2001), pp. 111-139.

25. J. P. Bérenger, "A perfectly matched layer for the absorption of electromagnetic waves," J. Comput. Phys. 114, 185-200 (1994).

26. J. P. Hugonin and P. Lalanne, "Perfectly matched layers as nonlinear coordinate transforms: a generalized formalization," J. Opt. Soc. Am. A 22, 1844-1849 (2005).

27. C. Vassallo, Optical Waveguide Concepts (Elsevier, 1991).

28. H. Raether, Surface Plasmons on Smooth and Rough Surfaces and on Gratings (Springer-Verlag, 1988).

29. A. W. Snyder and J. D. Love, Optical Waveguide Theory (Chapman \& Hall, 1983).

30. Ph. Lalanne, J. P. Hugonin, S. Astilean, M. Palamaru, and K. D. Möller, "One-mode model and Airy-like formulae for 1D metallic gratings," J. Opt. A Pure Appl. Opt. 2, 48-51 (2000).

31. This approximation amounts to considering that the slit is narrow enough so that all modes are evanescent except the fundamental one; see Ref. 30 for a discussion of this approximation with respect to the metallic film thickness $h$.

32. Standard techniques, like the adaptive Simpson quadrature method implemented with the function quad in MATLAB (MathWorks, Inc.), can be used.

33. H. Lochbihler and R. A. Depine, "Highly conducting wire gratings in the resonance domain," Appl. Opt. 32, 3459-3465 (1993). 